ARTICLE

Received 14 Apr 2016 | Accepted 23 Sep 2016 | Published 14 Nov 2016

DOl: $10.1038 /$ ncomms13329

OPEN

\title{
Akkermansia muciniphila mediates negative effects of IFN $\gamma$ on glucose metabolism
}

Renee L. Greer ${ }^{1, \star}$, Xiaoxi Dong ${ }^{2, \star}$, Ana Carolina F. Moraes ${ }^{3}$, Ryszard A. Zielke ${ }^{2}$, Gabriel R. Fernandes ${ }^{4}$, Ekaterina Peremyslova ${ }^{2}$, Stephany Vasquez-Perez ${ }^{1}$, Alexi A. Schoenborn ${ }^{5}$, Everton P. Gomes ${ }^{6}$, Alexandre C. Pereira ${ }^{6}$, Sandra R.G. Ferreira ${ }^{3}$, Michael Yao ${ }^{7}$, Ivan J. Fuss ${ }^{7}$, Warren Strober ${ }^{7}$, Aleksandra E. Sikora ${ }^{2}$, Gregory A. Taylor ${ }^{8}$, Ajay S. Gulati ${ }^{5}$, Andrey Morgun ${ }^{2, \star \star} \&$ Natalia Shulzhenko ${ }^{1, \star \star}$

Cross-talk between the gut microbiota and the host immune system regulates host metabolism, and its dysregulation can cause metabolic disease. Here, we show that the gut microbe Akkermansia muciniphila can mediate negative effects of IFN $\gamma$ on glucose tolerance. In IFN $\gamma$-deficient mice, $A$. muciniphila is significantly increased and restoration of IFN $\gamma$ levels reduces $A$. muciniphila abundance. We further show that IFN $\gamma$-knockout mice whose microbiota does not contain $A$. muciniphila do not show improvement in glucose tolerance and adding back $A$. muciniphila promoted enhanced glucose tolerance. We go on to identify Irgm1 as an IFN $\gamma$-regulated gene in the mouse ileum that controls gut $A$. muciniphila levels. A. muciniphila is also linked to IFN $\gamma$-regulated gene expression in the intestine and glucose parameters in humans, suggesting that this trialogue between IFN $\gamma$, A. muciniphila and glucose tolerance might be an evolutionally conserved mechanism regulating metabolic health in mice and humans.

\footnotetext{
${ }^{1}$ College of Veterinary Medicine, Oregon State University, 105 Dryden Hall, 450 SW 30th Street, Corvallis, Oregon 97331, USA. ${ }^{2}$ College of Pharmacy, Oregon State University, 1601 SW Jefferson Way, Corvallis, Oregon 97331, USA. ${ }^{3}$ Department of Epidemiology, School of Public Health, University of São Paulo, Av. Dr Arnaldo, 715, São Paulo, SP 01246-904, Brazil. ${ }^{4}$ Oswaldo Cruz Foundation, René Rachou Research Center, Av. Augusto de Lima, 1715, Belo Horizonte, MG 30190-002, Brazil. ${ }^{5}$ Division of Pediatric Gastroenterology, University of North Carolina at Chapel Hill, 260 MacNider Building, CB\# 7220, Chapel Hill, North Carolina 27599, USA. ${ }^{6}$ Laboratory of Genetics and Molecular Cardiology, Heart Institute (InCor), University of São Paulo Medical School, Av. Dr Eneas de Carvalho Aguiar, 44, São Paulo, SP 05403-000, Brazil. ${ }^{7}$ Mucosal Immunity Section, Laboratory of Immune Defenses, National Institute of Allergy and Infectious Diseases, Bethesda, Maryland 20892, USA. ${ }^{8}$ Geriatric Research, Education and Clinical Center, VA Medical Center, Departments of Medicine, Molecular Genetics and Microbiology and Immunology, Division of Geriatrics and Center for the Study of Aging and Human Development, Duke Box 3003, Duke University Medical Center, Durham, North Carolina 27710, USA. * These authors contributed equally to this work. ${ }^{\star \star}$ These authors jointly supervised this work. Correspondence and requests for materials should be addressed to A.M. (email: anemorgun@hotmail.com) or to N.S. (email: natalia.shulzhenko@oregonstate.edu).
} 
A $\mathrm{n}$ important advance of the last couple of decades in biomedical science is the recognition that mammalian organisms do not function as a collection of functionally independent systems. Rather, there is extensive cooperation among systems that is essential for life, and its absence can result in dysfunction and disease. Numerous studies have revealed the involvement of the immune system in regulation of metabolism, and how the alteration of the immune system can contribute to metabolic abnormalities such as type 2 diabetes and metabolic syndrome $e^{1-5}$. These studies have primarily focused on immune cell effects on fat, liver and muscle, as besides the pancreas, these tissues are considered major metabolic organs responsible for glucose and lipid metabolism. One such example is the influence of IFN $\gamma$, which is a central cytokine of the immune system, on systemic glucose metabolism. Previous studies have shown that mice deficient in IFN $\gamma$ have improved glucose tolerance ${ }^{6-8}$. Mechanistically, this phenomenon has been attributed to reduced hepatic glucose production ${ }^{6}$ and increased insulin sensitivity, possibly related to reduced adipose inflammation in case of obese animals ${ }^{8}$.

More recently, the gut has emerged as an important player in systemic metabolism. Besides producing several hormones, the gut harbours thousands of microbes (the gut microbiota) which themselves function as a metabolically active organ ${ }^{9,10}$. Therefore, by modulating the composition and dynamics of the gut microbiota, the immune system may ultimately exert a major impact on the metabolism of the organism. A few recent studies have demonstrated physiologically important trialogues among the immune system, gut microbiota and metabolism ${ }^{11-14}$. However, despite the emerging evidence of importance of such trialogues, much research continues to focus on two-component dialogues, thus failing to appreciate the complete picture of communication between multiple systems.

In the current study, we addressed whether the established dialogue between IFN $\gamma$ and glucose metabolism involves a third player-the gut microbiota. By using systems biology approaches and analysing transkingdom interactions we found that, indeed, the effect of IFN $\gamma$ on glucose tolerance is mediated by one of the members of mouse gut microbiota, A. muciniphila. Further, we have identified immunity-related GTPase family, $\mathrm{M}$ (Irgm1) as an IFN $\gamma$-regulated host gene responsible for control of $A$. muciniphila levels in the gut. In addition, the investigation of human subjects revealed that $A$. muciniphila may play similar roles in mouse and human physiology.

\section{Results}

IFN $\gamma$-regulated bacterial modulators of glucose metabolism. Similar to previous reports ${ }^{6-8}$, we observed that glucose tolerance is significantly improved in IFN $\gamma \mathrm{KO}$ mice (Fig. 1a). To start addressing our hypothesis that gut microbiota is a mediator of effect of IFN $\gamma$ on glucose metabolism, we first treated wild-type (WT) and IFN $\gamma \mathrm{KO}$ mice with a cocktail of antibiotics that has been successfully employed in previous studies to eliminate the majority of gut bacteria to test their role in host physiology ${ }^{15-17}$. Overall, glucose metabolism was improved following antibiotic treatment in both genotypes (Fig. 1a), which is consistent with previous findings that, as a whole, microbiota worsen glucose metabolism ${ }^{18-2}$. Importantly for this study, treatment with antibiotics abolished differences between the two genotypes, supporting our hypothesis that microbiota mediate the effect of IFN $\gamma$ on glucose metabolism (Fig. 1a). Body weight and food intake alone could not consistently explain differences in glucose tolerance (Supplementary Fig. 1).

We next sought to determine microbe(s) mediating effect of IFN $\gamma$ on glucose metabolism. Such microbes would need to fulfill two criteria: (1) to be regulated by IFN $\gamma$ and (2) to regulate glucose metabolism. Thus, in the exploratory phase, we first assessed which microbes were differentially abundant under IFN $\gamma$ perturbation. Next, in a separate set of analyses using correlations with metabolic measurements, we identified which of the IFN $\gamma$-regulated bacteria could be potential regulators of glucose metabolism (Fig. 1b). To identify such microbes and to minimize confounding effects, we used two independent methods to perturb IFN $\gamma$ levels-genetic disruption of IFN $\gamma$ and blockade with anti-IFN $\gamma$ antibody (Fig. 1b). When microbial abundances between IFN $\gamma \mathrm{KO}$ and corresponding wild-type mice were compared by sequencing of the bacterial $16 \mathrm{~S}$ ribosomal RNA (rRNA) gene, 555 differentially abundant operational taxonomic units (OTUs) were identified, corresponding to 33 different genera (Supplementary Fig. 2A, Supplementary Data 1). Next, to narrow and validate our findings, we used a second method to perturb IFN $\gamma$ levels. We took advantage of the fact that germfree mice have very low levels of systemic and intestinal IFN $\gamma$ and that microbiota induce expression of this cytokine in the gut ${ }^{22}$ (Supplementary Fig. 2E). We colonized wild-type germfree mice with microbiota from IFN $\gamma \mathrm{KO}$ mice and blocked the rising levels of IFN $\gamma$ with an anti-IFN $\gamma$ antibody to maintain low levels during colonization while a control group was treated with rat IgG (Supplementary Fig. 2E). We reasoned that taxa that have similar differential abundance in both experiments (genetic knockout and antibody blockade) are more likely to be regulated by IFN $\gamma$. As expected, we observed a significant increase in IFN $\gamma$ levels 7 days after colonization that was prevented by anti-IFN $\gamma$ antibody injection (Supplementary Fig. 2E). Sequencing of the $16 \mathrm{~S}$ rRNA in caecum revealed that 248 OTUs were differentially abundant (Supplementary Fig. 2D, Supplementary Data 2), of which 69 OTUs were concordant with the IFN $\gamma$ KO versus WT results (Fig. 1c, Supplementary Data 3).

Once we identified IFN $\gamma$-regulated bacteria, we searched for those that would be predicted to mediate the effect of the cytokine on glucose metabolism. To achieve this, we analysed correlations between the abundance of IFN $\gamma$-regulated microbes and glucose metabolism parameters such as fasted glucose levels and area under curve of glucose tolerance test (AUC-GTT). This analysis was performed in IFN $\gamma \mathrm{KO}$ mice so that direct effects of IFN $\gamma$ could not bias the correlation. With this approach ${ }^{23}$, microbial candidates that mediate the effect of IFN $\gamma$ on glucose metabolism should present a positive correlation with glucose levels and AUC-GTT if they are enriched in the presence of IFN $\gamma$, and negative correlation if they are depleted by IFN $\gamma$ (see experimental outline in Supplementary Fig. 3). Through this analysis we identified four different OTUs, all corresponding to A. muciniphila, as top candidate improvers of glucose metabolism (Fig. 1d). Increased abundance of $A$. muciniphila is detected in both the ileum and stool of IFN $\gamma \mathrm{KO}$ mice, and levels in the stool are representative of those in the ileum (Fig. 1e, Supplementary Fig. 2B,C) and correlate to fasting glucose and AUC-GTT (Fig. 1f,g). In addition, one OTU corresponding to Bacteroidetes S24-7, which could not be assigned to a specific taxon, was identified as a top candidate for worsening of glucose tolerance metrics (Fig. 1d).

A. muciniphila mediates effect of IFN $\gamma$ on glucose tolerance. A. muciniphila is a well-known, cultivable species present in both the mouse and human microbiota ${ }^{24}$. Interestingly, $A$. muciniphila has previously been linked to metabolism-it is reduced in obese mice and patients, and restoration of its levels improves glucose metabolism in mouse models of metabolic disease ${ }^{25-27}$. Our analysis, thus far, predicted $A$. muciniphila as a key candidate for improvement of glucose tolerance in lean IFN $\gamma \mathrm{KO}$ mice. To validate this relationship, we performed a series of confirmatory 
loss- and gain-of-function experiments (Fig. 2a). First, we restored IFN $\gamma$ levels in $\mathrm{KO}$ mice by administering exogenous recombinant IFN $\gamma$. All IFN $\gamma \mathrm{KO}$ mice showed identical initial glucose tolerance (Fig. 2b) at the start of the study. Following
2 weeks of injections, serum IFN $\gamma$ levels were elevated compared with PBS control, but did not reach wild-type levels; therefore it is unlikely that activation of IFN $\gamma$ pathways was induced above what would be expected for wild-type mice (Fig. 2f). Mice that
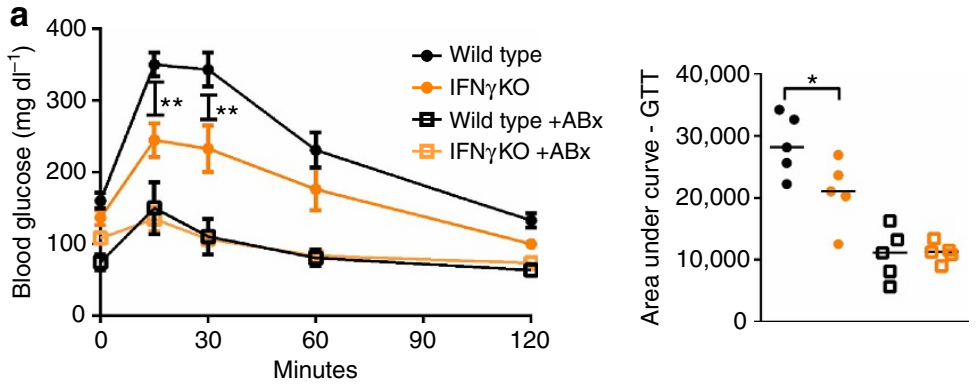

b Exploratory phase

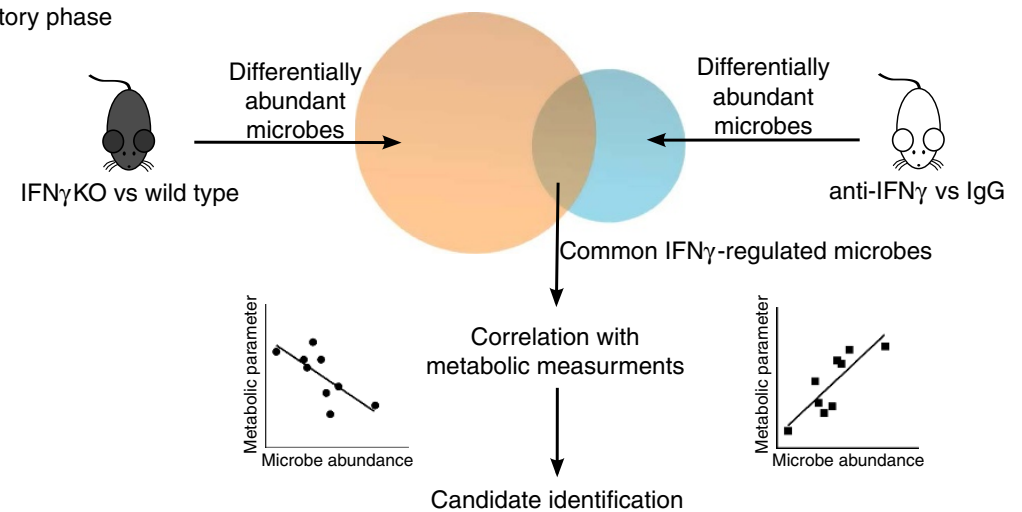

C
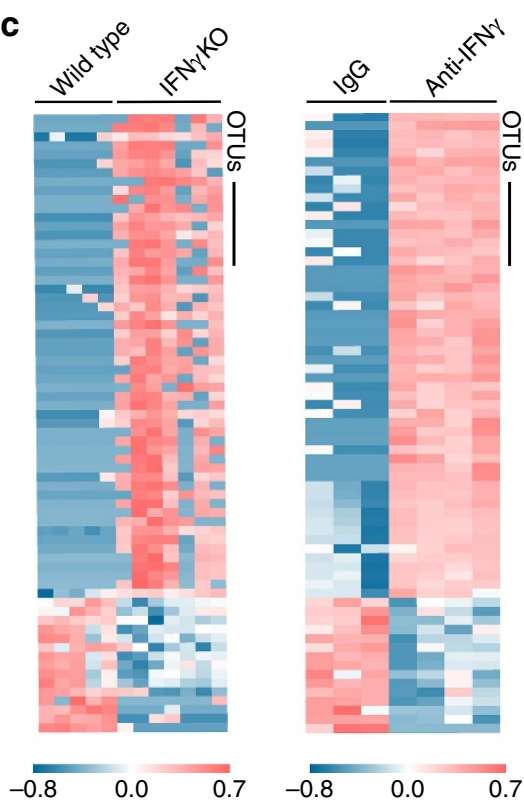

e

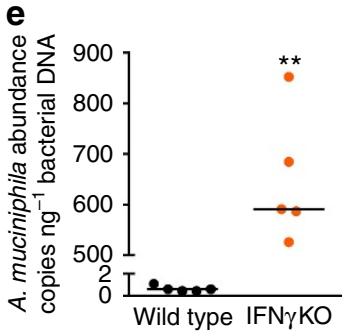

d 0.6

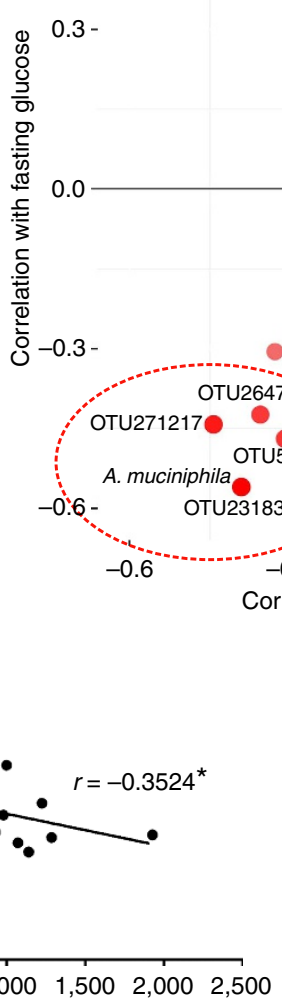

A. muciniphila (copies $\mathrm{ng}^{-1}$ bacterial DNA)

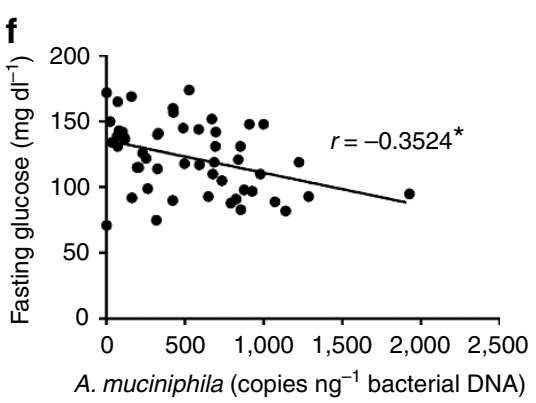

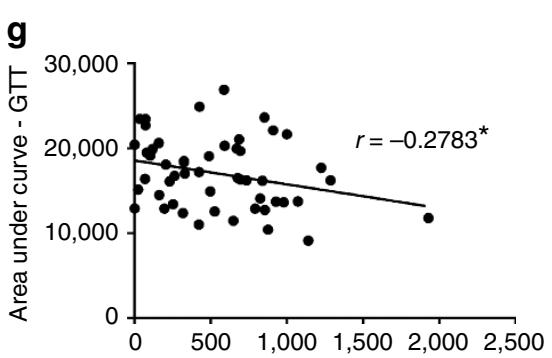

A. muciniphila (copies $\mathrm{ng}^{-1}$ bacterial DNA) 
received IFN $\gamma$ showed significantly worse glucose tolerance than PBS controls (Fig. 2c), coincident with a decrease in abundance of A. muciniphila levels (Fig. 2d). These data demonstrate the ability of IFN $\gamma$ to regulate $A$. muciniphila as well as to regulate glucose tolerance, but do not rule out the possibility that these two effects are independent.

Next, to directly test if IFN $\gamma$ acts through A. muciniphila as our predictive analysis suggests, we bred IFN $\gamma \mathrm{KO}$ mice with A. muciniphila-negative wild-type mice to generate A. muciniphila-negative IFN $\gamma$ heterozygotes, which were then interbred to ultimately obtain A. muciniphila-negative IFN $\gamma \mathrm{KO}$ mice $\left(\mathrm{IFN} \gamma \mathrm{KO} / A k k_{n e g}\right.$ ) that was possible due to lack of exposure from heterozygous parents (Fig. 2a middle panel; Supplementary Fig. 4D). After three generations of breeding, we achieved close to non-detectable levels ( $<1$ copy per ng bacterial DNA) of $A$. muciniphila in the stool of IFN $\gamma \mathrm{KO}$ mice (Supplementary Fig. 4D). There was no difference in glucose tolerance between wild-type and IFN $\gamma \mathrm{KO} / A k k_{\text {neg }}$ mice (Fig. 3b), demonstrating that by removal of $A$. muciniphila from the system we could abrogate the effect of IFN $\gamma$ on glucose levels. However, we reasoned that the breeding strategy might have altered the abundance of other taxa in gut microbiota in addition to A. muciniphila. Therefore we performed $16 \mathrm{~S}$ rRNA gene profiling of the IFN $\gamma \mathrm{KO} / A k k_{\text {neg }}$ microbiota compared with natively $A$. muciniphila positive mice from Jackson Labs. We identified only three taxa other than A. muciniphila to be different following this breeding strategy (Supplementary Table 1). Therefore, to test whether A. muciniphila, and not some other altered taxa, was causative of metabolic improvement in IFN $\gamma \mathrm{KO}$ mice, we reconstituted a subset of IFN $\gamma \mathrm{KO} / A k k_{n e g}$ mice with A. muciniphila (IFN $\gamma \mathrm{KO} /$ $A k k_{\text {pos }}$ ) (Fig. 3e). Seven days after colonization we observed better systemic glucose tolerance in IFN $\gamma \mathrm{KO} / A k k_{\text {pos }}$ mice, while IFN $\gamma \mathrm{KO}$ that did not receive $A$. muciniphila continued presenting glucose tolerance similar to wild-type mice (Fig. 3d, Supplementary Fig. 4), thus confirming that A. muciniphila is sufficient to mediate the effects of IFN $\gamma$ on systemic glucose metabolism. Finally, we restored IFN $\gamma$ levels in these IFN $\gamma \mathrm{KO} /$ $A k k_{n e g}$ and IFN $\gamma \mathrm{KO} / A k k_{\text {pos }}$ mice through injection of recombinant IFN $\gamma$. Only mice carrying A. muciniphila responded to treatment by worsening of glucose tolerance (compare Fig. $3 \mathrm{f}$ to Fig. 3h, Supplementary Fig. 4), thus demonstrating that IFN $\gamma$ acts by controlling A. muciniphila to worsen glucose tolerance in IFN $\gamma \mathrm{KO}$ mice. As previous studies primarily linked A. muciniphila to glucose metabolism in obese mice ${ }^{26,27}$, we also tested its ability to improve glucose metabolism in lean wild-type mice. Indeed, administration of $A$. muciniphila enhanced glucose tolerance in lean wild-type mice (Supplementary Fig. 5).

It is possible that the administration of A. muciniphila may have altered the abundance of other microbes that could, in turn, alter glucose tolerance. Therefore, we performed analysis to identify microbes that are potentially regulated by A. muciniphila and have evidence to be related to glucose metabolism. We identified three microbial genera that showed different abundance after A. muciniphila colonization and correlation to glucose tolerance, including Akkermansia, (False discovery rate $($ FDR $)<0.1$; Supplementary Table 2). A. muciniphila presented the strongest and most significant correlation. However, these other microbes might be interesting areas of further study. It is also possible that IFN $\gamma$ injection altered microbes in addition to A. muciniphila. Therefore we performed a similar analysis as above, comparing taxa abundance before and after injection within IFN $\gamma \mathrm{KO} / A k k_{p o s}$ mice. Although some minor trends of alteration of microbe abundance were observed, no genera except A. muciniphila were significantly altered by rIFN $\gamma$ injection at FDR $<0.1$. Therefore, although we cannot rule out a role for other microbes in mediating glucose tolerance upon administration of A. muciniphila and following injection of $\operatorname{rIFN} \gamma$, our analysis did not provide any plausible candidate that may play a role in glucose tolerance responses.

Irgm1 is a mediator of the effect of IFN $\gamma$ on A. muciniphila. Now that we have established A. muciniphila as a main contributor to improved glucose tolerance in IFN $\gamma \mathrm{KO}$ mice, the question remained how IFN $\gamma$ controls $A$. muciniphila levels. IFN $\gamma$ has a central role in orchestrating response to multiple gut microbes by driving different effector mechanisms ${ }^{28}$. To identify genes mediating effect of IFN $\gamma$ on A. muciniphila, we employed a comprehensive approach by measuring global gene expression. As a first step of our analysis we searched for mouse genes whose expression is regulated by IFN $\gamma$ in the ileum, but not dependent on the presence of A. muciniphila in the gut microbiota (that is, genes located downstream of IFN $\gamma$ and upstream of A. muciniphila). To detect these genes we compared ileal gene expression between wild-type, IFN $\gamma \mathrm{KO} / A k k_{\text {neg }}$ and IFN $\gamma \mathrm{KO} / A k k_{\text {pos }}$ mice. These analyses revealed 229 differentially expressed genes $(\mathrm{FDR}<0.1)$ between wild-type and IFN $\gamma \mathrm{KO}$ mice regardless of $A$. muciniphila status (Fig. 4a).

Network analysis has been an efficient tool in the identification of physiological processes and finding causal genes in host-microbe interactions ${ }^{15}$, as well as in cancer ${ }^{29,30}$. Therefore, we reconstructed a gene network of the IFN $\gamma$-dependent mouse ileum transcriptome which was comprised of 165 out 229 differentially expressed genes (Fig. 4b). As it could be expected, most of these genes had lower expression in IFN $\gamma \mathrm{KO}$ mice compared with controls. The interrogation of the network revealed overrepresentation of Gene Ontologies for immune responses including MHC (major histocompatibility complex) Class I antigen presentation, $\mathrm{T}$ cell activation and interferon-inducible GTPase (Supplementary Data 4). Furthermore, among top hub genes (high connectivity degree) that usually consist of upstream regulators were Stat1, Igtp, Tap1 and other genes representing the aforementioned immune pathways (Supplementary Data 4).

Often further investigation is focused on hub genes because of their potential probability to be master regulators of

\footnotetext{
Figure 1 | Identification of $\boldsymbol{A}$. muciniphila as a predicted IFN $\boldsymbol{\gamma}$-dependent regulator of glucose tolerance. (a) Intraperitoneal glucose tolerance test (IP-GTT) and area under the curve quantification in conventional IFN $\gamma$ KO and wild-type control mice before (closed circles) and after (open squares) 2 weeks of antibiotic cocktail treatment ( $n=5$ per group). Glucose tolerance curves shown as mean \pm s.e.m., median line is displayed on dot plots. (b) Experimental outline describing the exploratory phase for prediction of IFN $\gamma$-regulated microbes that are modulators of glucose metabolism. (c) Heat maps of common differentially abundant microbes in IFN $\gamma$ KO versus wild-type stool and anti-IFN $\gamma$ versus IgG caecal content. Differentially abundant microbes are selected based on $t$-test FDR $<0.1$. (d) Correlation of differentially abundant microbes to area under curve of glucose tolerance (AUC-GTT) test and fasting glucose. Colour intensity indicates direction of change of microbe in IFN $\gamma$ KO versus wild type (red $=$ more abundant in IFN $\gamma$ KO). Size of each point indicates Spearman correlation $P$ value with larger spots representing higher significance. Dashed circles indicate $P$ value cutoff of 0.05 . All four points within the red circle are unique OTUs, all representing A. muciniphila. (e) Quantification of $A$. muciniphila copy number by qPCR, represented as copies $A$. muciniphila genome per ng total 16S DNA. ( $n=5$ per group, one representative experiment out of 3). (f,g) Spearman correlation of $A$. muciniphila copies per ng bacterial DNA with fasting glucose $(\mathbf{f})$ and area under the curve of glucose tolerance test $(\mathbf{g})$ in IFN $\gamma \mathrm{KO}$ mice $(n=50) .{ }^{\star} P<0.05,{ }^{\star \star} P<0.01$, ${ }^{\star * \star} P<0.001$ by one-tailed Mann-Whitney test except where indicated otherwise.
} 

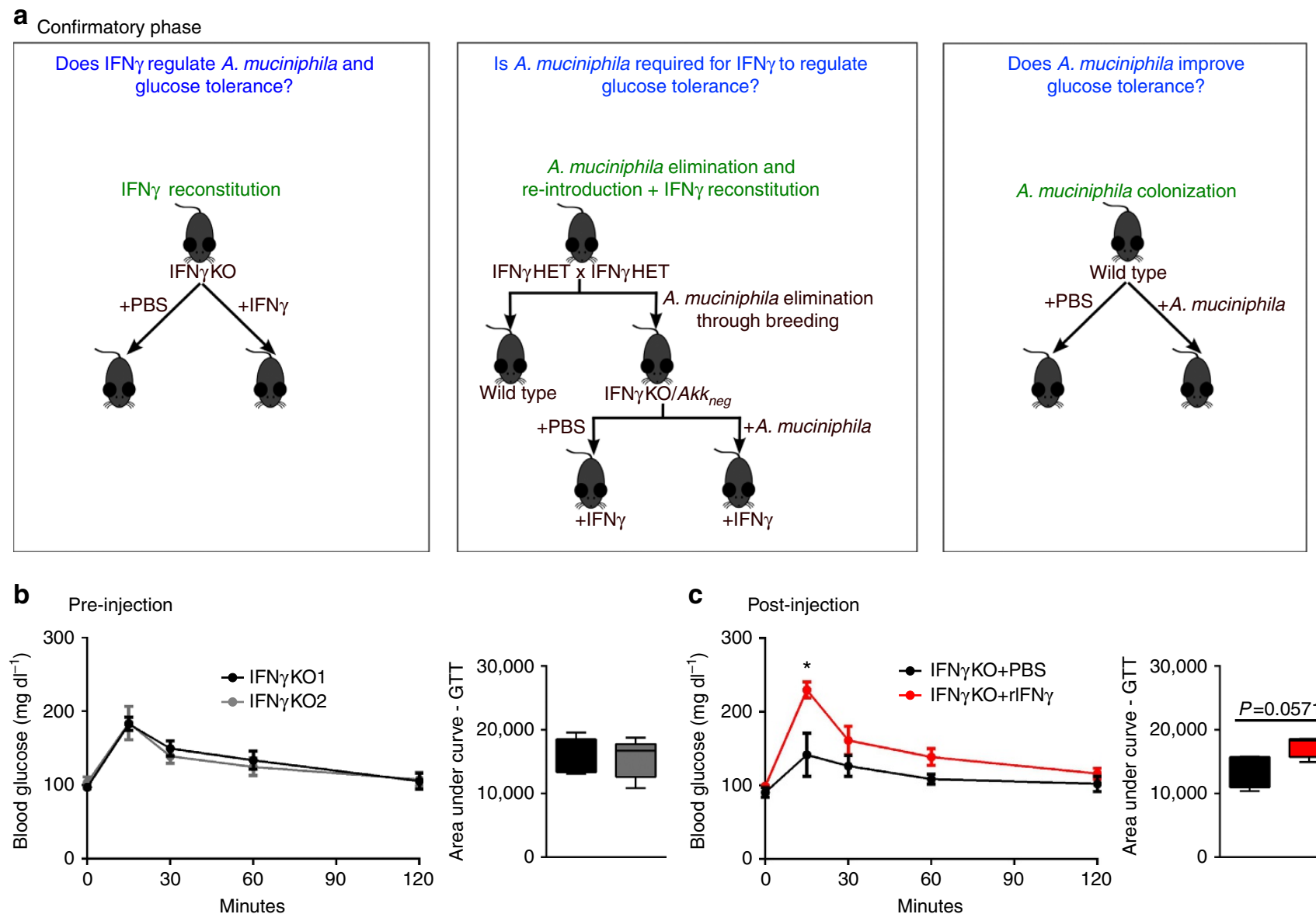
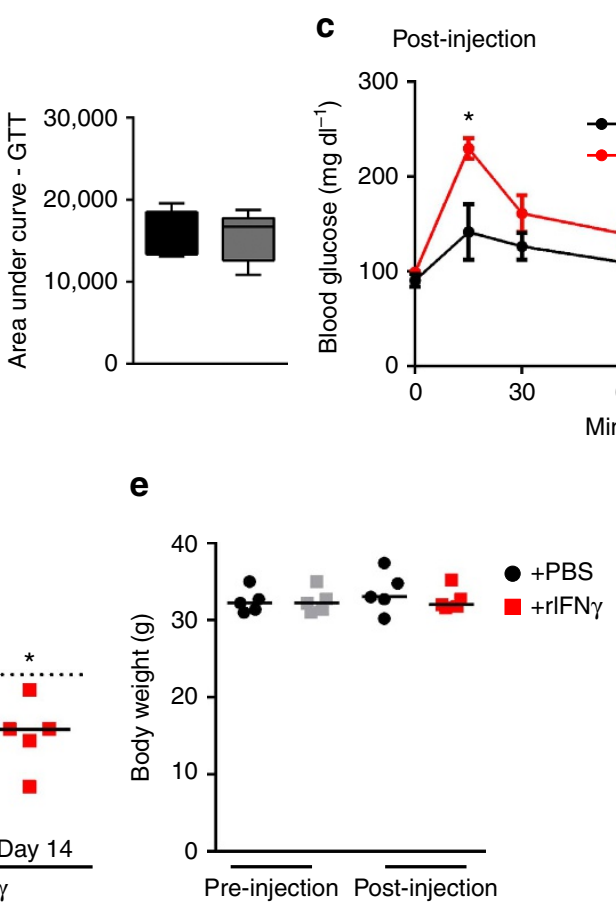

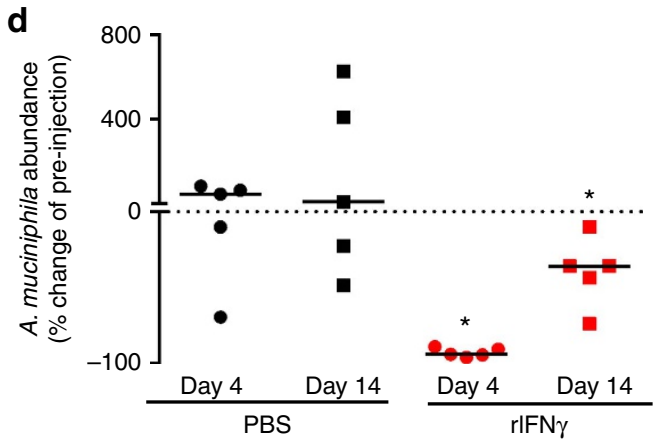

e $\mathbf{f}$

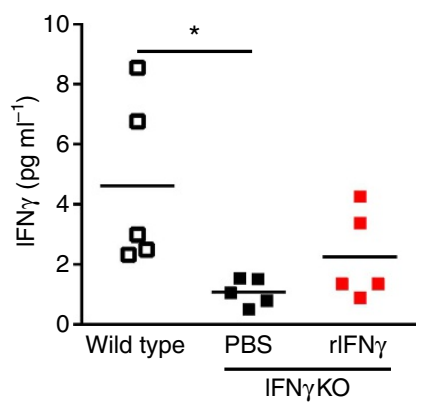

Figure 2 | IFN $\gamma$ reconstitution validates IFN $\gamma$ as a regulator of $\boldsymbol{A}$. muciniphila and glucose tolerance. (a) Experimental outline describing the confirmatory phase where the identified candidate from Fig. $1 \mathrm{~b}$ exploratory phase, A. muciniphila, is directly tested by three independent approaches. Readouts of all experiments are quantification of $A$. muciniphila abundance and assessment of glucose tolerance. (b,c) IP-GTT and area under the curve of IFN $\gamma$ KO mice before (b) and following 2 weeks of rIFN $\gamma$ or PBS administration (c). (d) A. muciniphila was quantified by qPCR. Shown is percent change of A. muciniphila abundance in stool from initial pre-injection levels after the 2-week injection period. (e) Body weight of all groups of mice pre- and post-injection. (f) Serum IFN $\gamma$ levels at the post-injection time point. Glucose tolerance curves shown as mean \pm s.e.m., box plots represent median with 25th and 75th percentile borders and error bars represent min-max. Median line is displayed on dot plots. For all glucose tolerance tests and qPCR results shown, $n=5$ per group. ${ }^{\star} P<0.05$ by one-tailed Mann-Whitney test.

processes $^{29,31}$. In this study, however, we were specifically interested in IFN $\gamma$-dependent genes positioned at the interface of the host gene regulatory network and A. muciniphila. To infer these genes, we again used causal inference analysis similar to that which was previously described for A. muciniphila discovery. In this analysis, we derived a ranking calculation that considered differential gene expression, correlation of each gene to A. muciniphila levels and peripheral-ness of a gene in the network (see 'Methods' section for complete details). This analysis revealed a few potential inhibitors of A. muciniphila, with Irgm1 being the top ranked candidate by this index (Fig. 4c,d). Next we tested the prediction of Irgm1 being an inhibitor of $A$. muciniphila by comparing abundance of this microbe between Irgm 1 knockout mice (Irgm1KO) and control mice in two different mouse facilities. Notably, despite a large difference in overall $A$. muciniphila abundance between the two sites, Irgm1KO mice had increased abundance of this microbe compared with their corresponding wild-type controls (Fig. 4e). To validate that this increase was due to the absence of Irgm1 and not a feedback loop altering IFN $\gamma$ 


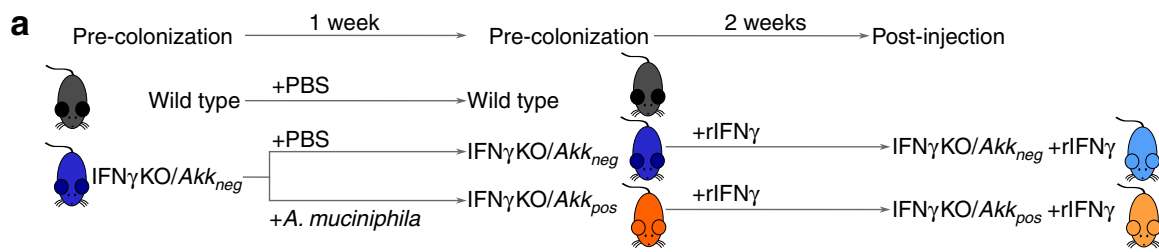

b Pre-colonization

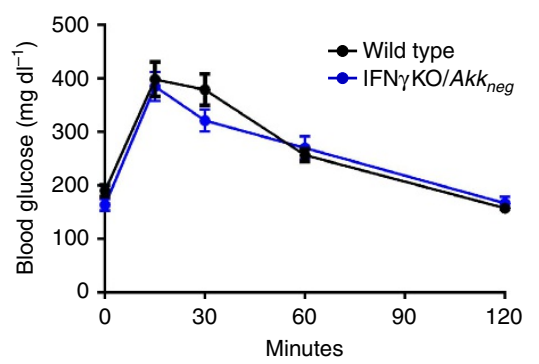

d Post-colonization

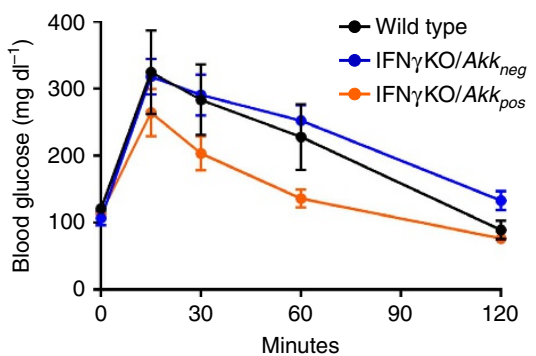

f Post-injection
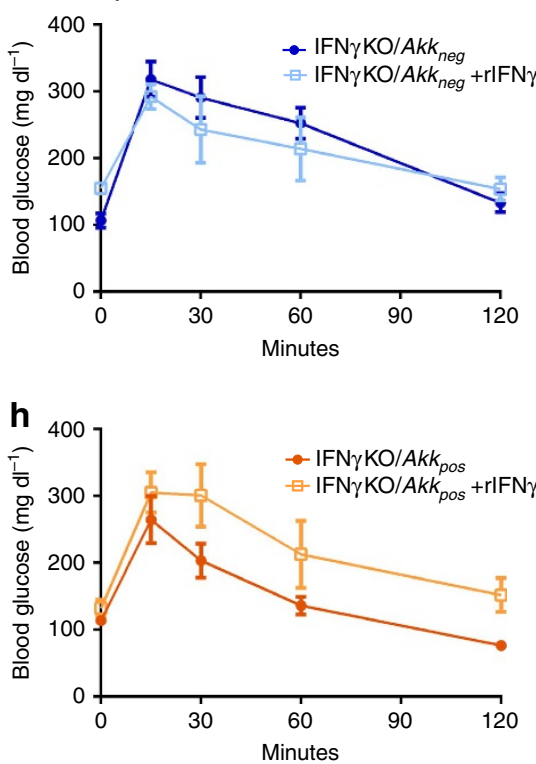

C
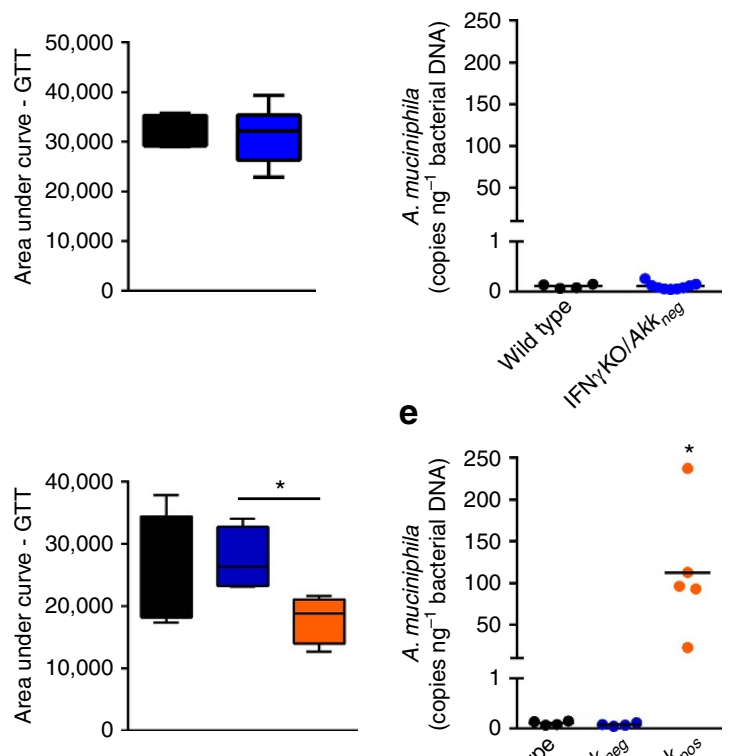

g
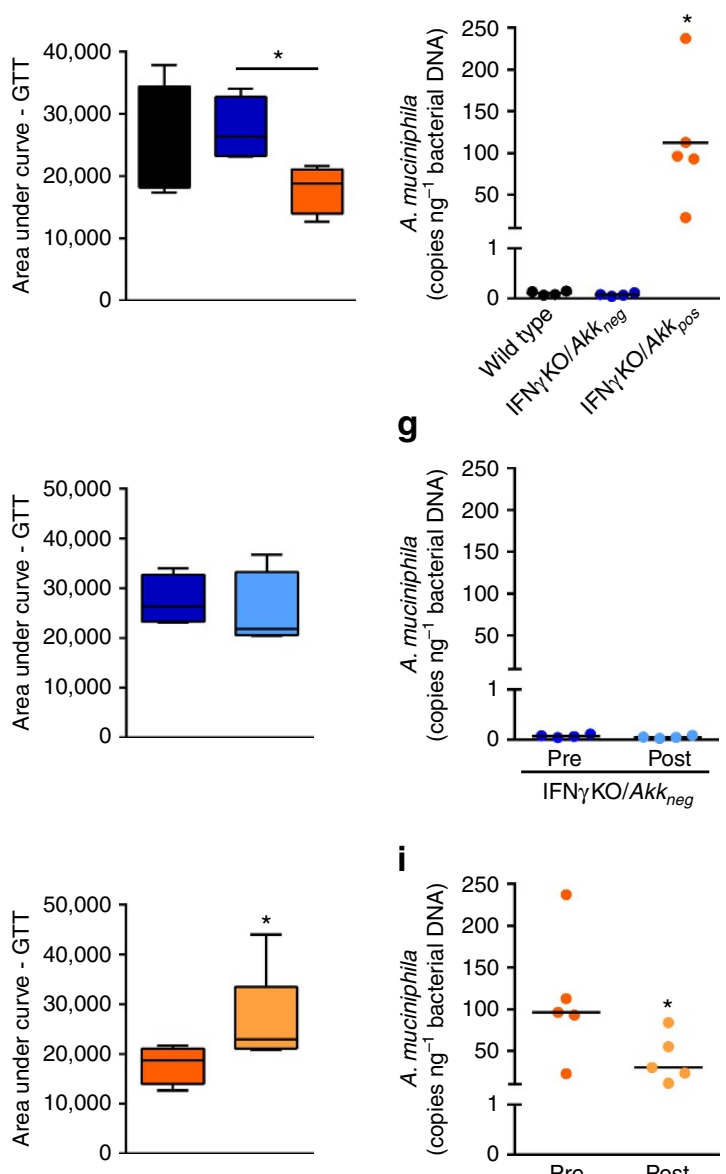

i

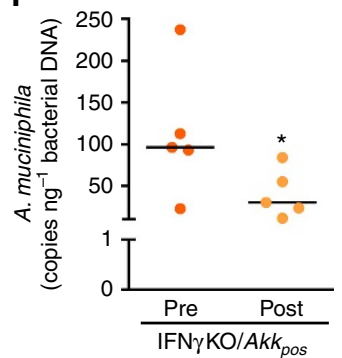

Figure 3 | A. muciniphila is required for IFN $\gamma$ regulation of glucose tolerance. (a) Experimental scheme: A. muciniphila-negative wild-type and IFN $\gamma$ KO mice were colonized with either PBS or A. muciniphila and subsequently injected with recombinant IFN $\gamma(\mathrm{rIFN} \gamma$ ). (b,d) Pre-colonization (b) and post-colonization (d) IP-GTT. (c,e) Pre-colonization (c) and post-colonization (e) A. muciniphila levels by qPCR expressed as copies of $A$. muciniphila per ng total bacterial DNA. (f,h) IP-GTT in IFN $\gamma K \mathrm{KO} / A k k_{\text {neg }}(\mathbf{f})$ and IFN $\gamma \mathrm{KO} / A k k_{\text {pos }}(\mathbf{h})$ before and after 2 weeks of injection with rIFN $\gamma$. Darker shades represent before injection and represent the same test shown in $\mathbf{d}$ for each respective group; lighter shades represent after injection. (g,i) $A$. muciniphila levels by qPCR expressed as copies of $A$. muciniphila per ng total bacterial DNA in IFN $\gamma \mathrm{KO} / A k k_{\text {neg }}(\mathbf{g})$ and IFN $\gamma \mathrm{KO} / A k k_{\text {pos }}$ (i) before and after 2 weeks of injection with rIFN $\gamma$. Darker shades represent before injection, lighter shades represent after injection. Glucose tolerance curves shown as mean \pm s.e.m., box plots represent median with 25th and 75th percentile borders and error bars represent min-max. Median line is displayed on dot plots. At pre-colonization time point $n=4$ for wild type and 9 for IFN $\gamma \mathrm{KO} / A k k_{\text {neg. }}$. At post-colonization and post-injection time points, $n=4$ for wild type and IFN $\gamma \mathrm{KO} / A k k_{n e g}$ and 5 for IFN $\gamma \mathrm{KO} / A k k_{\text {pos }}{ }^{\star} P<0.05$ by one-tailed Mann-Whitney test. 
signalling overall, we examined global gene expression in the ileum of these mice. Overall, very few genes from our previously identified IFN $\gamma$-dependent network were significantly altered (Supplementary Data 5). Notably, IFN $\gamma$ itself was not changed, nor were any of our top candidate A. muciniphila regulators from our previous network analysis (Fig. 4f). Thus, these results corroborate our computational prediction that Irgm1 is a significant factor in regulation of A. muciniphila by IFN $\gamma$.

A. muciniphila relates to glucose and IFN $\gamma$ in humans. A. muciniphila is also a frequent resident of the human gut microbiome ${ }^{24}$. Therefore, we took advantage of a cohort of subjects enrolled by Brazilian Advento Study Group to see if the

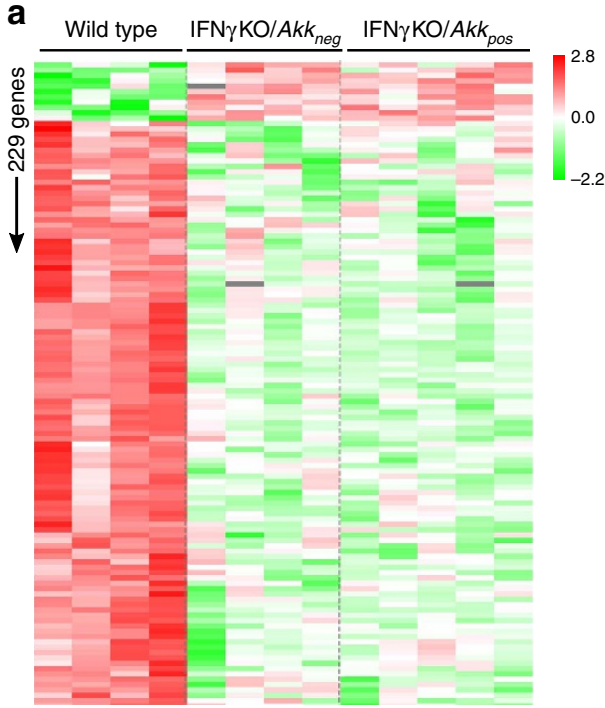

C

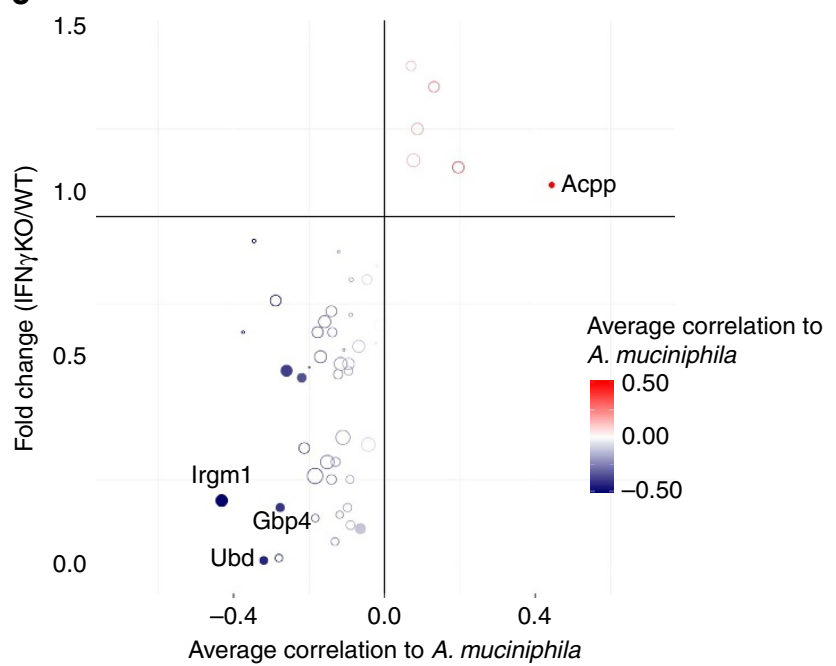

b

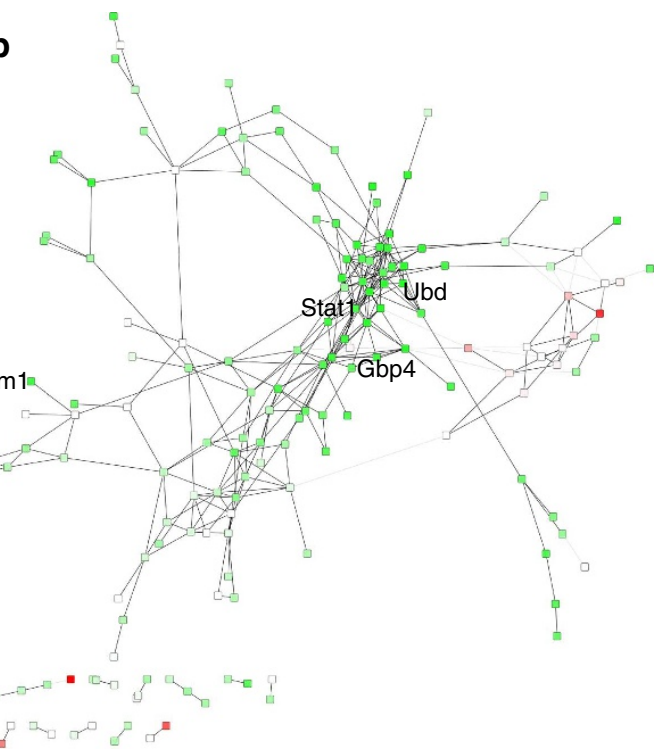

d

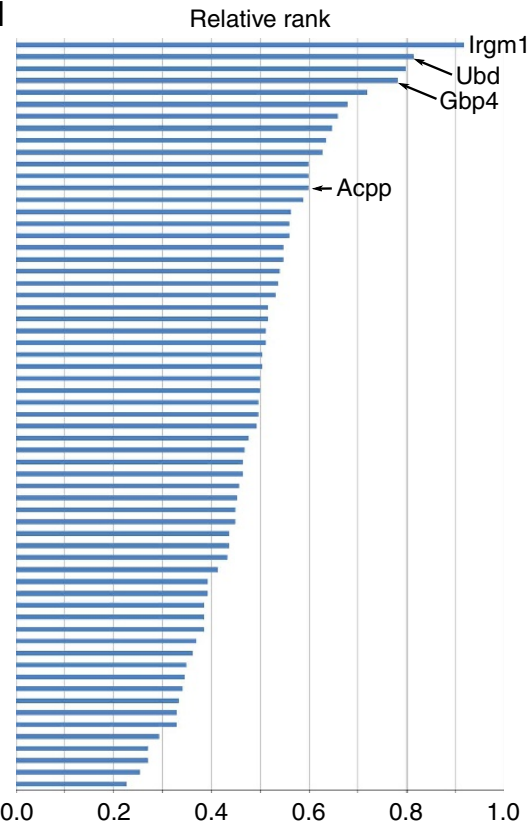

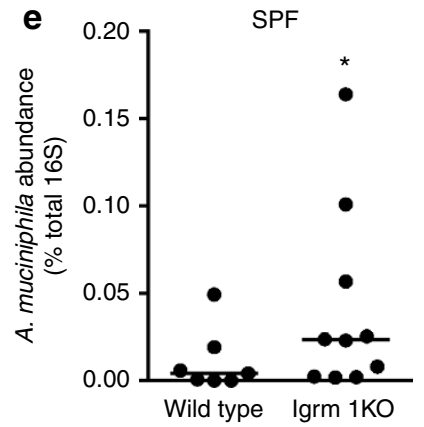
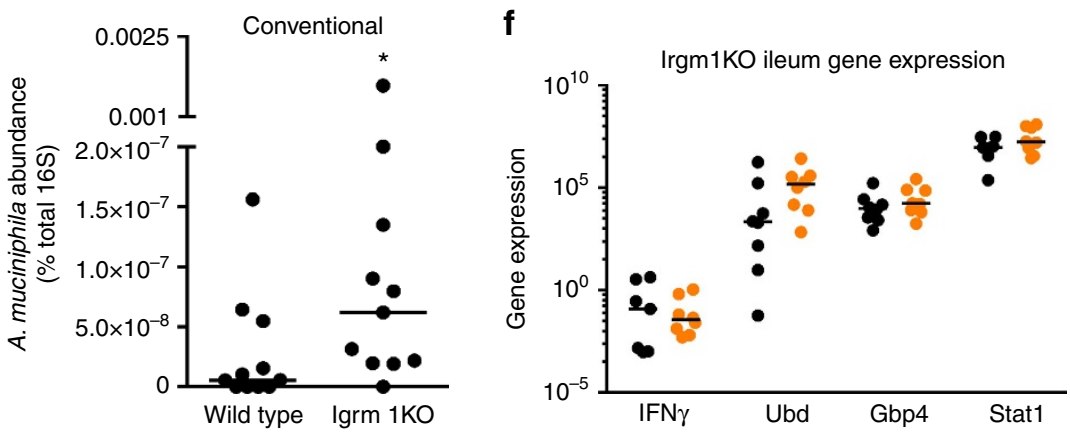
relation between A. muciniphila and metabolism we observed in mice can be also found in human population. Investigations have recently related levels of $A$. muciniphila with diabetes and/or obesity $^{25,32-34}$, however, several other metagenomic studies did not report an association between this bacterium and metabolic abnormalities in humans ${ }^{35,36}$. Considering that multiple gut microbes besides $A$. muciniphila may influence glucose metabolism, we speculated that in cases where this microbe is at low levels, it is less likely to contribute considerably to the phenotype because other more abundant microbes would be stronger players. To define biologically significant levels, we referred back to our IFN $\gamma \mathrm{KO}$ mice that presented negative correlation between $A$. muciniphila and fasting glucose levels (Fig. 1f,g). The abundance of $A$. muciniphila was $>1 \%$ in the majority of those mice (Supplementary Fig. 2). Therefore, from the total of 295 human subjects we selected those with abundance of $A$. muciniphila $\geq 1 \%(N=94)$. We found that $A$. muciniphila had a weak but significant negative correlation with glucose and glycated haemoglobin (HbA1c) (Spearman $r=-0.3167$ $P<0.001$ and $r=-0.3033 P<=0.01$, respectively) (Fig. 5a,b). We then used American Diabetes Association guidelines 37,38 for classification of these subjects into three groups based on glycaemia status by fasting plasma glucose, $2 \mathrm{~h}$ plasma glucose (2-PG) and HbAlc and assessed A. muciniphila abundance in these groups. Individuals with normal glucose metabolism showed significantly higher $A$. muciniphila abundance compared with type 2 diabetics, with pre-diabetics showing an intermediate abundance of A. muciniphila (Fig. 5c). In the group with diabetes, some patients were on treatment with metformin, which had been previously associated with increased A. muciniphila in mice 27,39 . However, we did not detect differences for A. muciniphila abundance, fasting glucose or HbAlc between subjects treated or not treated with metformin (Supplementary Fig. 6). While these results require a confirmation in independent human cohorts, they support the idea that A. muciniphila may play a similar role in mice and humans in regulation of glucose metabolism.

Data regarding intestinal expression of IFN $\gamma$ was not available in human subjects that were evaluated for faecal microbiome and glucose metabolism. Therefore, we turned to another group of human subjects in whom we had measured global gene expression and A. muciniphila levels in duodenal biopsies. This group of subjects consisted of three subgroups including healthy volunteers, and two different groups of patients with common variable immunodeficiency. Analysis showed a trend to a negative correlation (Pearson $r \approx-0.3, P=0.127$ ) between IFN $\gamma$ gene expression and A. muciniphila levels (Fig. 5d, top gene). Therefore, we decided to analyse the human gene signature corresponding to mouse homologues we have defined as stimulated by IFN $\gamma$ in the murine intestine (Fig. 4b). Out of about 220 mouse genes, we found 162 human homologues with 141 of them being detectable in duodenal biopsies.

Analysing the correlation between expression of these genes and $A$. muciniphila levels, we found that approximately half of the gene signature (69 genes) had the same signs of correlations in all three analysed groups of subjects. These were all negative correlations with no gene presenting a consistent (through all three groups) positive correlation (Fig. $5 \mathrm{~d}$, test for one proportion $P<0.0001$, Supplementary Data 6). Thus, despite small sample sizes in each individual group, the combined analysis showed consistent negative correlation for several IFN $\gamma$-dependent genes supporting the hypothesis that IFN $\gamma$ may contribute to control of A. muciniphila levels not only in mice, but also in humans.

\section{Discussion}

Our study has uncovered a missing link between IFN $\gamma$ and glucose metabolism by demonstrating that a gut commensal, A. muciniphila, is a key microbe responsible for improved glucose tolerance observed in IFN $\gamma \mathrm{KO}$ mice (Fig. 5e). Notably, two primary players that have been revealed to mediate the effect of IFN $\gamma$ (Irgm1 and A. muciniphila) could not have been easily predicted based solely on the existing knowledge in the field. Rather, the generation of testable hypotheses in both cases was mainly a result of causal inference involving trankingdom network analysis that we have recently developed (reviewed in ref. 40). This approach has been previously successful in finding microbes and microbial genes that affect host phenotype ${ }^{15}$. This is the first time, however, when such strategy aided in prediction of host gene controlling a specific member of gut microbiota.

It is well established that IFN $\gamma$ is important for control of multiple, primarily intracellular, pathogens. The effect of this cytokine on gut microbiota, however, has not been explored. Using two methods (genetic deletion and blocking antibody) we revealed that multiple OTUs from commensal microbiota were affected by IFN $\gamma$ (Fig. 1, Supplementary Data 1-2). Following identification of IFN $\gamma$-regulated microbes, causal inference analysis allowed us to discern candidates relevant to the phenotype of interest (that is, glucose levels). We then validated the prediction that $A$. muciniphila is a mediator of effect of IFN $\gamma$ on glucose metabolism by colonizations of different hosts with A. muciniphila and reconstitution of IFN $\gamma \mathrm{KO}$ mice with recombinant IFN $\gamma$.

Altogether, the colonization of IFN $\gamma \mathrm{KO}$ and wild-type mice with $A$. muciniphila shows that that this bacterium can improve glucose metabolism (fasting glucose and glucose tolerance) in different hosts. We cannot, however, make a definitive conclusion which other microbes might be required for its effect on glucose metabolism.

\footnotetext{
Figure 4 | IFN $\gamma$ regulates A. muciniphila abundance through Irgm1. (a) Heat map of transcript abundance of IFN $\gamma$-dependent genes. Genes that show differential abundance between wild type and IFN $\gamma$ KO ( $t$-test FDR $<0.1)$, but no difference between IFN $\gamma K O / A k k n e g$ and IFN $\gamma K O / A k k p o s ~(t$-test FDR $<0.1)$ are shown. (b) Network reconstruction of IFN $\gamma$-dependent genes shown in $\mathbf{a}$. Colours indicate fold change of expression as indicated in $\mathbf{a}$. A file containing complete information for this network is available for download upon request. (c) Correlation of IFN $\gamma$-dependent genes with $A$. muciniphila levels. Pearson correlation between ileum A. muciniphila abundance and gene expression were calculated in three groups separately and the average correlation coefficient was shown. Colour intensity of each point indicates strength of correlation to $A$. muciniphila levels. Size of each point indicates average shortest path length, with larger points representing longer paths. (d) Ranking of IFN $\gamma$-dependent genes as potential regulators of $A$. muciniphila. Ranking takes into account strength of correlation with $A$. muciniphila and average shortest path length, with longer path lengths (that is, more peripheral to the network) resulting in higher ranking scores. See 'Methods' section for a more detailed description of calculation of this rank score. (e) A. muciniphila abundance in Irgm1KO mice housed in specific pathogen free conditions ( $n=7$ wild type, $10 \mathrm{Irgm} 1 \mathrm{KO})$ and conventional conditions $(n=11$ per genotype) by qPCR, represented as per cent A. muciniphila of total 16S rRNA DNA. (f) Gene expression of top IFN $\gamma$-dependent candidate genes from d determined by RNA-seq in the Irgm1KO ileum under specific pathogen free conditions; $n=7$ wild type (black symbols), 10 Irgm1KO (orange symbols). Acpp, acid phosphatase, prostate; Gbp4, guanylate binding protein 4; Irgm1, immunity-related GTPase family, M; Stat1, signal transducer and activator of transcription 1; SPF, Specific pathogen free; Ubd, Ubiquitin D. Median line displayed on dot plots. ${ }^{\star} P<0.05$ by one-tailed Mann-Whitney test.
} 

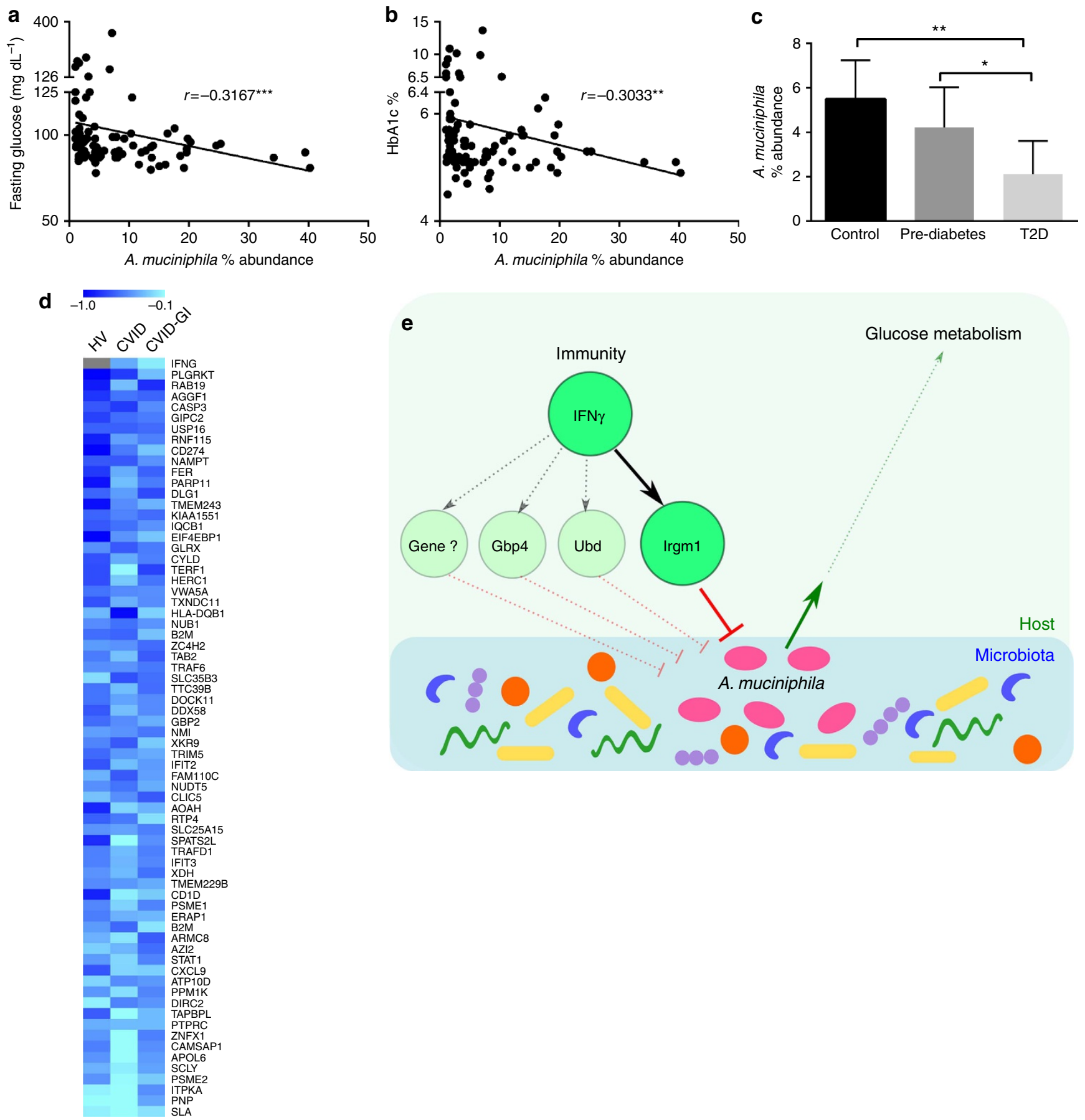

Figure 5 | A. muciniphila correlates to glucose measures in human subjects and is reduced in diabetic patients. (a,b) Spearman correlation of A. muciniphila percent abundance with fasting glucose (a) and HbA1c (b) in participants in the Advento Study $(n=94)$ (c) A. muciniphila percent abundance in normal $(n=58)$, pre-diabetic $(n=31)$ and type 2 diabetic subjects $(n=11)$. Bar plot represents mean and $95 \%$ confidence interval. Significance assessed by one-tailed Mann-Whitney test. (d) Heat map of Pearson correlation coefficients between each individual IFN $\gamma$-dependent gene and abundance of $A$. muciniphila of duodenal biopsies in three groups of samples. Individual $P$ value $<0.2$, combined FDR $<0.1$ for 59 out of 69 genes (Supplementary Data 6); genes ranked by strength of correlation according to Fisher's combined probability test. Grey colour indicates that a gene was below the level of detection. ${ }^{\star} P<0.05,{ }^{\star \star} P<0.01,{ }^{\star \star \star} P<0.001$. (e) Graphical model for regulation of glucose metabolism by IFN $\gamma$ through the microbiota. IFN $\gamma$ regulates expression of genes such as Irgm1 and Gbp4, which in turn, contribute to regulation of $A$. muciniphila levels in the gut. Differences in A. muciniphila abundance ultimately result in differences in systemic glucose tolerance in the host, with higher abundance of $A$. muciniphila inducing improvement of tolerance. CVID, Common Variable Immunodeficiency; CVID-GI, CVID with gastrointestinal symptoms; HV, healthy volunteer.

Although, in the current study we did not investigate which type of immune cells are of the source(s) IFN $\gamma$, intraepithelial T lymphocytes are the most plausible candidates. Besides their ability to produce IFN $\gamma$, intraepithelial T lymphocytes are the strongest responders among cells of adaptive immune system to changes in the microbiota ${ }^{15}$. This agrees with a recent study demonstrating that A. muciniphila levels are higher in mice deficient of $\mathrm{T}$ and $\mathrm{B}$ lymphocytes (Rag1KO) than in wild-type mice ${ }^{41}$. 
We also inferred and validated a molecule downstream of IFN $\gamma$, Irgm1, as a regulator of A. muciniphila. Although Irgm 1 has been previously implicated in the control of intracellular pathogens ${ }^{42,43}$, Irgm1KO mice also have Paneth cell abnormalities ${ }^{44}$. Because the secretion of antimicrobial proteins from Paneth cells is induced by IFN $\gamma$ (ref. 45), we can speculate that Irgm1 may be a part of this signalling cascade. Ultimately, impaired production of antimicrobial peptides in the gut of IFN $\gamma \mathrm{KO}$ mice may be a potential mechanism leading to outgrowth of $A$. muciniphila.

The second most favourable candidate among those identified as host genes-regulators of $A$. muciniphila is ubiquitin $\mathrm{D}$ ( $U b d$ or FAT10; Fig. 4c,d), Interestingly, disruption of Ubd in mice has been shown to improve glucose tolerance along with other metabolic parameters but the impact on gut microbiota has not been examined ${ }^{46}$. Thus, while we have shown that $\operatorname{Irgm} 1$ is a mediator of the effect of IFN $\gamma$ on A. muciniphila, it is plausible that other IFN $\gamma$-dependent mechanisms may also contribute to this phenomenon.

Our results from human subjects demonstrate that A. muciniphila regulation of metabolism may be an evolutionally conserved mechanism between mice and humans. Relevance of the trialogue (IFN $\gamma \rightarrow$ A. muciniphila $\rightarrow$ glucose metabolism) to human health is further supported by evidence of increased levels of IFN $\gamma$ producing cells in diabetes ${ }^{47,48}$ and decrease abundance of $A$. muciniphila $25,32-34$ in obese and diabetic patients. Interestingly, A. muciniphila levels have been recently demonstrated to negatively correlate with several inflammation markers associated with metabolic disease in mice ${ }^{49}$. Overall, these results suggest that loss of this bacterium can be due to local immune activation in the gut during disease and that this loss has implications for systemic metabolism. This topic warrants further investigation that should involve comprehensive evaluation of patients' immune status including in intestinal tissues.

Our findings may also explain response of mice to metformin, the most widely used drug for type 2 diabetes, that was also shown to block IFN $\gamma$ production ${ }^{50}$ and to increase levels of A. muciniphila in mice ${ }^{27}$. However, this particular mechanism might be different in mice and humans because neither our data (Supplementary Fig. 6) nor other more comprehensive human studies ${ }^{51}$ found association between $A$. muciniphila and treatment with metformin.

Finally our study revealed a new homeostatic regulatory process in mammalian organisms, where a member of different kingdom, A. muciniphila, constitutes an integral part of the interaction between the supposedly functionally distinct and distant systems of immunity and glucose metabolism. Furthermore, our results and other published work in mouse models and human subjects suggest that this transkingdom interaction may be common in mammals ${ }^{25-27}$. Over the years, biologists have drawn boundaries between systems and kingdoms. Our results highlight the fact that these boundaries must be crossed to fully understand the complexity of living organisms.

\footnotetext{
Methods

Mice. IFN $\gamma$ knockout (IFN $\gamma \mathrm{KO}$ on C57BL/6J background) and C57BL/6J controls were initially purchased from The Jackson Laboratory (Bar Harbor, Maine). Mice were housed at the Laboratory Animal Resource Center at Oregon State University under standard 12-h light cycle with free access to food (5001, Research Diets) and water. For all colonization studies, mice were maintained with autoclaved supplies, food (5010, Research Diets) and water. Adult mice of 8-10 weeks were used for all studies. Male mice were used for metabolic experiments, while males and females were used for microbiota sequencing and gene expression experiments. For experiments with IFN $\gamma \mathrm{KO} / A k k_{n e g}$ mice, C57BL/6J and IFN $\gamma \mathrm{KO}$ originally purchased from Jackson Labs were bred to generate heterozygous IFN $\gamma \mathrm{HET}$ mice. There heterozygous mice were then interbred for two generations to IFN $\gamma \mathrm{KO} /$
} $A k k_{n e g}$ mice. Experimental procedures were carried out in accordance with protocols approved by the Oregon State University Institutional Animal Care and Usage Committee. Antibiotics were administered in drinking water for 2 weeks in the following concentrations: ampicillin $\left(\mathrm{gl}^{-1}\right)$, vancomycin $\left(0.5 \mathrm{gl}^{-1}\right)$, neomycin trisulfate $\left(\mathrm{gl}^{-1}\right)$ and metronidazole $\left(1 \mathrm{gl}^{-1}\right)$. Irgm $1 \mathrm{KO}$ mice generated and maintained at the Durham VA and Duke University Medical Centers in conventional and specific pathogen free colonies. These mice have been described previously ${ }^{42,43}$ and were backcrossed to $\mathrm{C} 57 \mathrm{Bl} / 6 \mathrm{NCr} 1$ mice for nine generations. Use of the Irgm1 mice was approved by the IACUC of the Durham VA and Duke University Medical Centers.

Bacterial culture. A. muciniphila ATCC BAA- 835 was streaked out from $-80^{\circ} \mathrm{C}$ on BD Brain Heart Infusion (BHI) agar supplemented with $0.4 \%$ mucin (Sigma) and incubated under anaerobic conditions using the GasPack 100 system (BD Biosciences) at $37^{\circ} \mathrm{C}$ for $36 \mathrm{~h}$. Bacterial colonies were swabbed from the plates, suspended in liquid $\mathrm{BHI}$ medium and $100 \mu \mathrm{l}$ of the solution was plated on BHI agar containing $0.4 \%$ mucin. After $36 \mathrm{~h}$ of incubation at $37^{\circ} \mathrm{C}$ in anaerobic jar, bacteria were swabbed from plates, suspended in $10 \mathrm{ml}$ of BHI containing $15 \%$ glycerol, aliquoted and stored at $-80^{\circ} \mathrm{C}$. To determine the colony forming units, one aliquot was thawed, serially diluted and plated on BHI agar, and bacterial colonies were enumerated after $36 \mathrm{~h}$.

Anti-IFN $\gamma$ and recombinant IFN $\gamma$ treatments. For anti-IFN $\gamma$ treatment, $100 \mu \mathrm{g}$ anti-IFN $\gamma$ (Clone R4-6A2, Oregon Health and Science University Monoclonal Antibody Core) or IgG control (Sigma-Aldrich) was injected intraperitoneally every 3 days. For recombinant IFN $\gamma$ treatment, $250 \mathrm{ng}$ carrier-free recombinant mouse IFN $\gamma$ (BioLegend) was injected intraperitoneally every other day.

Glucose tolerance testing. Mice were fasted for $6 \mathrm{~h}$ during the light phase with free access to water. A concentration of $2 \mathrm{mg} \mathrm{kg}^{-1}$ glucose (Sigma-Aldrich) was injected intraperitoneally. Blood glucose was measured at 0 (immediately before glucose injection), 15, 30, 60 and $120 \mathrm{~min}$ with a Freestyle Lite glucometer (Abbot Diabetes Care).

Food intake monitoring. Mice were housed individually. Food weights were recorded every other day over a period of 1 week (four individual measurements), and average intake per day for each 2-day period was determined and averaged over the week measurement period for each individual.

Bacterial DNA extraction and quantitative PCR. For microbial DNA, frozen faecal pellets, caecal content and whole ileum with content were resuspended in $1.4 \mathrm{ml}$ ASL buffer (Qiagen) and homogenized with $2.8 \mathrm{~mm}$ ceramic beads followed by $0.5 \mathrm{~mm}$ glass beads using an OMNI Bead Ruptor (OMNI International). DNA was extracted from the entire resulting suspension using QiaAmp mini stool kit (Qiagen) according to manufacturer's protocol. DNA was quantified using Qubit broad range DNA assay (Life Technologies). A total of $10 \mathrm{ng}$ of DNA was used for quantitative PCR (qPCR) reactions for A. muciniphila (AM1: 5'CAGCACGTGA AGGTGGGGAC', AM2: $5^{\prime}$ CCTTGCGGTTGGCTTCAGAT) ${ }^{52}$ and total bacteria (UniF340: 5'ACTCCTACGGGAGGCAGCAGT, UniR514: 5'ATTACCGCGG CTGCTGGC) $^{53}$. qPCR was performed using Fast SYBR master mix (Applied Biosystems) and StepOne Plus Real Time PCR system and software (Applied Biosystems).

RNA preparation and gene expression analysis. RNA was extracted using an OMNI Bead Ruptor and $2.8 \mathrm{~mm}$ ceramic beads (OMNI International) in RLT buffer followed by Qiashredder and RNeasy kit using Qiacube (Qiagen) automated extraction according to manufacturer's specifications. Total RNA was quantified using Nanodrop (Thermo Scientific) ${ }^{54}$. Complementary DNA was prepared using iScript reverse transcription kit (Bio-Rad) and qPCR was performed using QuantiFast SYBR mix (Qiagen) and StepOne Plus Real Time PCR system and software (Applied Biosystems).

$16 \mathrm{~S}$ rRNA gene sequencing and taxonomic analysis. The V4 region of $16 \mathrm{~s}$ rRNA gene was amplified using universal primers $(515 \mathrm{f} \text { and } 806 \mathrm{r})^{55}$. Individual samples were barcoded, pooled to construct the sequencing library, and then sequenced using an Illumina Miseq (Illumina, San Diego, CA) to generate pair-ended $250 \mathrm{nt}$ reads. The raw forward-end fastq reads were quality-filtered, demultiplexed and analysed using 'quantitative insights into microbial ecology' (QIIME) ${ }^{56}$. For quality filtering, the default parameters of QIIME were maintained in which reads with a minimal Phred quality score of $<20$, containing ambiguous base calls and containing fewer than $187 \mathrm{nt}$ (75\% of $250 \mathrm{nt}$ ) of consecutive high-quality base calls, were discarded. Additionally, reads with three consecutive low-quality bases were truncated. The samples sequenced were demultiplexed using 12 bp barcodes, allowing 1.5 errors in the barcode. UCLUST (http://www.drive5.com/uclust) ${ }^{57}$ was used to choose OTUs with a threshold of $97 \%$ sequence similarity against Green gene database (version gg_12_10) ${ }^{58}$. A representative set of sequences from each OTU were selected for taxonomic identification of each OTU by selecting the cluster seeds. The Greengenes OTUs 
(version gg_12_10) reference sequences (97\% similarity) were used for taxonomic assignment using BLAST ${ }^{59}$ with $E_{-}$value 0.001 . Raw read counts of OTUs were normalized against total number of reads that passed quality filtration to generate relative abundance of OTUs. Differentially abundant OTUs were identified using univariate $t$-test in BRB array tools' 'class comparison between groups of arrays' module. BRB Array Tools was developed by the Biometric Research Branch of the National Cancer Institute under the direction of R. Simon (http://linus.nci.nih.gov/ BRB-ArrayTools.html).

RNA-seq. RNA libraries were prepared with Wafergen Biosystems PrepX RNA-Seq Sample and Library Preparation Kits for the Apollo 324 NGS Library Prep System and sequenced using Illumina HiSeq. Forward read sequences with at least one ambiguous nucleotide were filtered out by prinseq ${ }^{60}$. Trimmomatic ${ }^{61}$ was used to trim Illumina adaptor sequences (parameters: seed mismatches:1, palindrome clip threshold: 10 and simple clip threshold: 10), to remove leading and trailing low-quality bases (below quality 3 ), to scan the read with a 4-base wide sliding window, and to cut when the average quality per base drops below 20 and to drop reads that below 60 bases long. Reads were aligned to mouse genome and transcriptome (ENSEMBLE release-70) using Tophat ${ }^{62}$ with default parameters. Number of reads per million for mouse genes were counted using HTSeq ${ }^{63}$ and differential abundance of genes that are detected in at least three samples were analysed using edge $\mathrm{R}^{64}$.

Network analysis and causal inference. The gene-gene network was reconstructed using following steps. First, for each pair of genes, four Pearson correlation coefficients and $P$ values are calculated using expression levels of each of the four groups of mouse samples on different genetic backgrounds $(\mathrm{C} 57 \mathrm{BL} / 6(n=8) \text {, Swiss-Webster }(n=7), \mathrm{B} 10 \mathrm{~A}(n=9) \text { and } \mathrm{BALB} / \mathrm{c}(n=10))^{15}$. Second, Fisher combined $P$ value is calculated from four $P$ values using Fisher's combined probability test. FDR value is calculated from the combined $P$ value, that is, we calculate a test statistic as

$$
\chi_{2 k}^{2} \sim-2 \sum_{i=1}^{k} \ln \left(P_{i}\right)
$$

where $k=$ number of groups ( 4 in this example) and $P_{i}$ is the $P$ value of a single group (one mouse strain in this example). A $P$ value (the combined $P$ value) for $\chi^{2}$ is calculated under the fact that it follows a chi-squared distribution with $2 k$ degrees-of-freedom. Third, the network is generated by selection and inclusion of gene-gene pairs as has been previously described ${ }^{40}$. Briefly, criteria for inclusion of gene-gene pairs are the following: Individual $P$ value of correlation within each group is $<0.3$; combined fisher $P$ value of all groups $<0.01$; the sign of correlation coefficients in four mouse strain groups should be consistent (all positive correlation or all negative correlation) and should be consistent with fold change relationship between the two genes (see 'Methods' section in ref. 23).

To prioritize genes that potentially mediate the effect of IFN $\gamma$ on A. muciniphila, we integrated four sources of ranking information: Absolute value of the average correlation coefficient of ileal gene expression with ileal A. muciniphila abundance across three groups of IFN $\gamma \mathrm{KO}$ mice; average shortest path length in gene-gene network (Fig. 4b); absolute value of $\log 10$ transformed fold change between their expression level in IFN $\gamma \mathrm{KO}$ versus wild-type control mice; directional matching of correlation across three IFN $\gamma \mathrm{KO}$ mouse groups, that is: assign positive sign to the absolute average correlation coefficient if the correlation signs are the same between all three groups, otherwise, assign negative sign to the absolute average correlation coefficient. Ranks from all four sources (corresponding values from smallest to largest) are summed to generate the final ranking of the genes.

Human data from the ADVENTO study. Subjects and protocol. A group of participants of the study Analysis of Diet and Lifestyle for Cardiovascular Prevention in Seventh-Day Adventists (ADVENTO-http://www.estudoadvento.org) conducted at University of São Paulo, Brazil was included in this cross-sectional study. The first 300 participants aged 35-65 years old were evaluated according to the eligibility criteria. Those with body mass index $\geq 40 \mathrm{~kg} \mathrm{~m}^{-2}$, history of inflammatory bowel diseases or persistent diarrhoea (longer than 2 weeks) and use of antibiotics or probiotic or prebiotic supplements within the 2 months before the data collection were not included. Five individuals were excluded from the final sample due to incomplete data. The University of São Paulo institutional ethics committee approved the study and written consent was obtained. Individuals were examined at the Investigation Center of University Hospital. After overnight fasting, they underwent a 2-h 75-g oral glucose tolerance test and American Diabetes Association criteria were used to define categories of glucose tolerance ${ }^{37,38}$.

Analytical measurements. Blood samples were immediately centrifuged and analysed. Plasma glucose was measured by the hexokinase method (ADVIA Chemistry; Siemens, Deerfield, IL, USA) and HBAlc by high-pressure liquid chromatography (Bio-Rad Laboratories, Hercules, CA, USA).

Gut microbiota. Faecal samples were maintained under refrigeration $\left(6^{\circ} \mathrm{C}\right)$ within a maximum of $24 \mathrm{~h}$ after collection, when the aliquots were stored at $-80^{\circ} \mathrm{C}$ until analysis. DNA was extracted using the Maxwell 16 DNA purification kit and the protocol carried out in the Maxwell 16 Instrument according to the manufacturer's instructions (Promega, Madison, WI, USA). Library preparation and sequencing were performed as described above for mouse samples. For analysis, high-quality sequences from 16S rRNA gene were obtained after trimming using Trimmomatic ${ }^{61}$. The paired reads were merged using FLASH $^{65}$ to reconstruct the contiguous sequenced region. Merged reads were then submitted to QIIME OTU picking pipeline ${ }^{56}$. The first step is the closed reference OTU clustering, using the GreenGenes ${ }^{66}$ version 13.5. This same database was used to assign taxonomic classification to each OTU.

Human data from the common variable immunodeficiency cohort. Subjects and protocol. Duodenal biopsy samples were collected from healthy volunteers $(n=4)$ and immunodeficient patients with $(n=7)$ or without gastrointestinal syndrome $(n=7)$ at the National Institutes of Health Clinical Center. All protocol and consent procedures were approved by the National Institute of Allergy and Infectious Diseases and Oregon State University Institutional Review Boards. The diagnosis of common variable immunodeficiency and the associated enteropathy were made following international guidelines ${ }^{67,68}$.

RNA isolation and sequencing. Total RNA was isolated using AllPrep kit (Qiagen) and prepared for sequencing using ScriptSeq Complete Gold Kit (Illumina). Four samples were pooled per lane and sequenced using paired end $100 \mathrm{bp} \mathrm{Illumina}$ Hiseq2000. Processing of raw data and analyses were performed as for mouse RNAseq except that reads were aligned with human transcriptome. For 16S rRNA gene sequencing, complementary DNA was prepared from RNA with SuperScript VILO kit (ThermoFisher), then amplified and sequenced using the same protocol as for mouse samples.

Correlation analysis. For analysis of correlation between A. muciniphila abundance and gene expression, we employed Pearson correlation in each one of the three patient groups followed by Fisher's combined probability test corrected by false discovery rate. To estimate a chance of given number of genes been negatively or positively correlated with A. muciniphila, we employed the test for one proportion implemented in https://www.medcalc.org/.

Statistical analysis. Statistical tests used for each comparison are described within corresponding figure legends. For large-scale data (transcriptome, microbiome and network reconstruction), statistical tests are described in the corresponding experimental procedures.

Data availability. Raw and processed data files for RNA-seq experiments have been deposited in ArrayExpress under accession code E-MTAB-3633. 16S rRNA gene sequencing raw read data have been deposited in the European Nucleotide Archive under accession codes PRJEB9551.

\section{References}

1. Donath, M. Y. \& Shoelson, S. E. Type 2 diabetes as an inflammatory disease. Nat. Rev. Immunol. 11, 98-107 (2011).

2. Nishimura, S. et al. CD8 + effector T cells contribute to macrophage recruitment and adipose tissue inflammation in obesity. Nat. Med. 15, 914-920 (2009).

3. Lumeng, C. N., Bodzin, J. L. \& Saltiel, A. R. Obesity induces a phenotypic switch in adipose tissue macrophage polarization. J. Clin. Invest. 117, 175-184 (2007).

4. Feuerer, M. et al. Lean, but not obese, fat is enriched for a unique population of regulatory T cells that affect metabolic parameters. Nat. Med. 15, 930-939 (2009).

5. Duffaut, C., Galitzky, J., Lafontan, M. \& Bouloumie, A. Unexpected trafficking of immune cells within the adipose tissue during the onset of obesity. Biochem. Biophys. Res. Commun. 384, 482-485 (2009).

6. Wong, N. et al. Deficiency in interferon-gamma results in reduced body weight and better glucose tolerance in mice. Endocrinology 152, 3690-3699 (2011).

7. Rocha, V. Z. et al. Interferon-gamma, a Th1 cytokine, regulates fat inflammation: a role for adaptive immunity in obesity. Circ. Res. 103, 467-476 (2008).

8. O'Rourke, R. W. et al. Systemic inflammation and insulin sensitivity in obese IFN-gamma knockout mice. Metabolism 61, 1152-1161 (2012).

9. Backhed, F. et al. The gut microbiota as an environmental factor that regulates fat storage. Proc. Natl Acad. Sci. USA 101, 15718-15723 (2004).

10. Turnbaugh, P. J. et al. An obesity-associated gut microbiome with increased capacity for energy harvest. Nature 444, 1027-1131 (2006).

11. Vijay-Kumar, M. et al. Metabolic syndrome and altered gut microbiota in mice lacking Toll-like receptor 5. Science 328, 228-231 (2010).

12. Caricilli, A. M. et al. Gut microbiota is a key modulator of insulin resistance in TLR 2 knockout mice. PLoS Biol. 9, e1001212 (2011).

13. Shulzhenko, N. et al. Crosstalk between B lymphocytes, microbiota and the intestinal epithelium governs immunity versus metabolism in the gut. Nat. Med. 17, 1585-1593 (2011). 
14. Everard, A. et al. Intestinal epithelial MyD88 is a sensor switching host metabolism towards obesity according to nutritional status. Nat. Commun. 5, 5648 (2014).

15. Morgun, A. et al. Uncovering effects of antibiotics on the host and microbiota using transkingdom gene networks. Gut 64, 1732-1743 (2015).

16. Iida, N. et al. Commensal bacteria control cancer response to therapy by modulating the tumor microenvironment. Science 342, 967-970 (2013).

17. Rakoff-Nahoum, S., Paglino, J., Eslami-Varzaneh, F., Edberg, S. \& Medzhitov, R. Recognition of commensal microflora by toll-like receptors is required for intestinal homeostasis. Cell 118, 229-241 (2004).

18. Caesar, R. et al. Gut-derived lipopolysaccharide augments adipose macrophage accumulation but is not essential for impaired glucose or insulin tolerance in mice. Gut 61, 1701-1707 (2012)

19. Hwang, I. et al. Alteration of gut microbiota by vancomycin and bacitracin improves insulin resistance via glucagon-like peptide 1 in diet-induced obesity. FASEB J. 29, 2397-2411 (2015).

20. Carvalho, B. M. et al. Modulation of gut microbiota by antibiotics improves insulin signalling in high-fat fed mice. Diabetologia 55, 2823-2834 (2012).

21. Membrez, M. et al. Gut microbiota modulation with norfloxacin and ampicillin enhances glucose tolerance in mice. FASEB J. 22, 2416-2426 (2008).

22. El Aidy, S. et al. Temporal and spatial interplay of microbiota and intestinal mucosa drive establishment of immune homeostasis in conventionalized mice. Mucosal Immunol. 5, 567-579 (2012).

23. Yambartsev, A. et al. Unexpected links reflect the noise in networks. Biol. Direct. doi: 10.1186/s13062-016-0155-0 (2016).

24. Derrien, M., Collado, M. C., Ben-Amor, K., Salminen, S. \& de Vos, W. M. The Mucin degrader Akkermansia muciniphila is an abundant resident of the human intestinal tract. Appl. Environ. Microbiol. 74, 1646-1648 (2008).

25. Karlsson, C. L. et al. The microbiota of the gut in preschool children with normal and excessive body weight. Obesity 20, 2257-2261 (2012).

26. Everard, A. et al. Cross-talk between Akkermansia muciniphila and intestinal epithelium controls diet-induced obesity. Proc. Natl Acad. Sci. USA 110, 9066-9071 (2013).

27. Shin, N. R. et al. An increase in the Akkermansia spp. population induced by metformin treatment improves glucose homeostasis in diet-induced obese mice. Gut 63, 727-735 (2014).

28. Janeway, C. Immunobiology: The Immune System in Health and Disease 6th edn (Garland Science, 2005).

29. Mine, K. L. et al. Gene network reconstruction reveals cell cycle and antiviral genes as major drivers of cervical cancer. Nat. Commun. 4, 1806 (2013).

30. Akavia, U. D. et al. An integrated approach to uncover drivers of cancer. Cell 143, 1005-1017 (2010).

31. Gerstein, M. B. et al. Architecture of the human regulatory network derived from ENCODE data. Nature 489, 91-100 (2012).

32. Santacruz, A. et al. Gut microbiota composition is associated with body weight, weight gain and biochemical parameters in pregnant women. Br J. Nutr. 104, 83-92 (2010).

33. Teixeira, F. S. et al. Faecal levels of Bifidobacterium and Clostridium coccoides but not plasma lipopolysaccharide are inversely related to insulin and HOMA index in women. Clin. Nutr. 32, 1017-1022 (2013).

34. Zhang, X. et al. Human gut microbiota changes reveal the progression of glucose intolerance. PLoS ONE 8, e71108 (2013).

35. Qin, J. et al. A metagenome-wide association study of gut microbiota in type 2 diabetes. Nature 490, 55-60 (2012).

36. Karlsson, F. H. et al. Gut metagenome in European women with normal, impaired and diabetic glucose control. Nature 498, 99-103 (2013).

37. American Diabetes Association. Classification and diagnosis of diabetes. Diabetes Care 38, S8-S16 (2015).

38. de Almeida-Pititto, B. et al. Type 2 diabetes in Brazil: epidemiology and management. Diabetes Metab. Syndr. Obes. 8, 17-28 (2015).

39. Lee, H. \& Ko, G. Effect of metformin on metabolic improvement and gut microbiota. Appl. Environ. Microbiol. 80, 5935-5943 (2014).

40. Dong, X. et al. Reverse enGENEering of regulatory networks from big data: a roadmap for biologists. Bioinform. Biol. Insights 61, 61-74 (2015).

41. Zhang, H., Sparks, J. B., Karyala, S. V., Settlage, R. \& Luo, X. M. Host adaptive immunity alters gut microbiota. ISME J. 9, 770-781 (2015).

42. Taylor, G. A. IRG proteins: key mediators of interferon-regulated host resistance to intracellular pathogens. Cell. Microbiol. 9, 1099-1107 (2007).

43. Collazo, C. M. et al. Inactivation of LRG-47 and IRG-47 reveals a family of interferon gamma-inducible genes with essential, pathogen-specific roles in resistance to infection. J. Exp. Med. 194, 181-188 (2001).

44. Liu, B. et al. Irgm1-deficient mice exhibit Paneth cell abnormalities and increased susceptibility to acute intestinal inflammation. Am. J. Physiol. Gastrointest. Liver Physiol. 305, G573-G584 (2013).

45. Farin, H. F. et al. Paneth cell extrusion and release of antimicrobial products is directly controlled by immune cell-derived IFN- $\gamma$. J. Exp. Med. 211, 1393-1405 (2014).
46. Canaan, A. et al. Extended lifespan and reduced adiposity in mice lacking the FAT10 gene. Proc. Natl Acad. Sci. USA 111, 5313-5318 (2014).

47. Sumarac-Dumanovic, M. et al. Therapeutic improvement of glucoregulation in newly diagnosed type 2 diabetes patients is associated with a reduction of IL-17 levels. Immunobiology 218, 1113-1118 (2013).

48. Pacifico, L. et al. Increased T-helper interferon-gamma-secreting cells in obese children. Eur. J. Endocrinol. 154, 691-697 (2006).

49. Schneeberger, M. et al. Akkermansia muciniphila inversely correlates with the onset of inflammation, altered adipose tissue metabolism and metabolic disorders during obesity in mice. Sci. Rep. 5, 16643 (2015).

50. Nath, N. et al. Metformin attenuated the autoimmune disease of the central nervous system in animal models of multiple sclerosis. J. Immunol. 182, 8005-8014 (2009).

51. Forslund, K. et al. Disentangling type 2 diabetes and metformin treatment signatures in the human gut microbiota. Nature 528, 262-266 (2015).

52. Collado, M. C., Derrien, M., Isolauri, E., de Vos, W. M. \& Salminen, S. Intestinal integrity and Akkermansia muciniphila, a mucin-degrading member of the intestinal microbiota present in infants, adults, and the elderly. Appl. Environ. Microbiol. 73, 7767-7770 (2007).

53. Amann, R. I. et al. Combination of $16 \mathrm{~S}$ rRNA-targeted oligonucleotide probes with flow cytometry for analyzing mixed microbial populations. Appl. Environ. Microbiol. 56, 1919-1925 (1990).

54. Du, P., Kibbe, W. A. \& Lin, S. M. Lumi: a pipeline for processing Illumina microarray. Bioinformatics 24, 1547-1548 (2008).

55. Caporaso, J. G. et al. Ultra-high-throughput microbial community analysis on the Illumina HiSeq and MiSeq platforms. ISME J. 6, 1621-1624 (2012).

56. Caporaso, J. G. et al. QIIME allows analysis of high-throughput community sequencing data. Nat. Methods 7, 335-336 (2010).

57. Edgar, R. C. Search and clustering orders of magnitude faster than BLAST. Bioinformatics 26, 2460-2461 (2010).

58. DeSantis, T. Z. et al. Greengenes, a chimera-checked 16S rRNA gene database and workbench compatible with ARB. Appl. Environ. Microbiol. 72, 5069-5072 (2006).

59. Altschul, S. F. et al. Gapped BLAST and PSI-BLAST: a new generation of protein database search programs. Nucleic Acids Res. 25, 3389-3402 (1997).

60. Schmieder, R. \& Edwards, R. Quality control and preprocessing of metagenomic datasets. Bioinformatics 27, 863-864 (2011).

61. Bolger, A. M., Lohse, M. \& Usadel, B. Trimmomatic: a flexible trimmer for Illumina sequence data. Bioinformatics 30, 2114-2120 (2014).

62. Trapnell, C., Pachter, L. \& Salzberg, S. L. TopHat: discovering splice junctions with RNA-Seq. Bioinformatics 25, 1105-1111 (2009).

63. Anders, S., Pyl, P. T. \& Huber, W. HTSeq--a Python framework to work with high-throughput sequencing data. Bioinformatics 31, 166-169 (2014).

64. Robinson, M. D., McCarthy, D. J. \& Smyth, G. K. edgeR: a bioconductor package for differential expression analysis of digital gene expression data. Bioinformatics 26, 139-140 (2010).

65. Magoc, T. \& Salzberg, S. L. FLASH: fast length adjustment of short reads to improve genome assemblies. Bioinformatics 27, 2957-2963 (2011).

66. McDonald, D. et al. An improved Greengenes taxonomy with explicit ranks for ecological and evolutionary analyses of bacteria and archaea. ISME J. 6, 610-618 (2011).

67. Mannon, P. J. et al. Excess IL-12 but not IL-23 accompanies the inflammatory bowel disease associated with common variable immunodeficiency. Gastroenterology 131, 748-756 (2006).

68. Bonilla, F. A. et al. International Consensus Document (ICON): common variable immunodeficiency disorders. J. Allergy Clin. Immunol. Pract. 4, 38-59 (2016).

\section{Acknowledgements}

This research was supported by startup funds for AM and NS from Oregon State University (OSU), USA; NIH U01 AI109695 (AM) and R01 DK103761 (NS) and by the Intramural Research Program of the NIH, NIAID (MY, IJF, WS). We also thank the FAPESP (São Paulo Research Foundation) for financial support (12/12626-9 and 12/ 03880-9) of ADVENTO Study. We thank Oregon State University Center for Genome Research and Biocomputing (CGRB) for sequencing services and technical support; Dr Daniel Cawley and the Oregon Health and Science University Monoclonal Antibody Core for production of IFN $\gamma$ antibodies; and the personnel of Oregon State University Laboratory Animal Resources Center.

\section{Author contributions}

A.M. and N.S. conceived the original idea, designed and supervised the studies and analysis, and wrote the manuscript. R.L.G. conceived the original idea, designed the studies, performed experiments, analysed the data and wrote the manuscript. X.D. designed the studies, performed microbiome and host transcriptome analyses, performed 
causal inference analysis and wrote the manuscript. S.V.-P. conducted and assisted with mouse experiments. E.P. prepared libraries and provided other technical assistance. R.A.Z. and A.E.S. prepared A. muciniphila and participated in writing of the manuscript. A.C.F.M., G.R.F., E.P.G., A.C.P. and S.R.G.F. recruited human subjects of ADVENTO study, conducted clinical and microbiome analysis and participated in writing the manuscript. M.Y., I.J.F., W.S. recruited patients, conducted clinical evaluations, performed duodenal biopsy collection. A.A.S., G.A.T. and A.S.G. performed analysis of Irgm $1 \mathrm{KO}$ mice and participated in writing of the manuscript.

\section{Additional information}

Supplementary Information accompanies this paper at http://www.nature.com/ naturecommunications

Competing financial interests: The authors declare no competing financial interests.

Reprints and permission information is available online at http://npg.nature.com/ reprintsandpermissions/
How to cite this article: Greer, R. L. et al. Akkermansia muciniphila mediates negative effects of IFN $\gamma$ on glucose metabolism. Nat. Commun. 7, 13329 doi: 10.1038/ ncomms13329 (2016).

Publisher's note: Springer Nature remains neutral with regard to jurisdictional claims in published maps and institutional affiliations.

(c) (i) This work is licensed under a Creative Commons Attribution 4.0 International License. The images or other third party material in this article are included in the article's Creative Commons license, unless indicated otherwise in the credit line; if the material is not included under the Creative Commons license, users will need to obtain permission from the license holder to reproduce the material. To view a copy of this license, visit http://creativecommons.org/licenses/by/4.0/

(C) The Author(s) 2016 